\title{
Jet Bundles and the Formal Theory of Partial Differential Equations
}

\author{
Richard Baker and Chris Doran \\ Astrophysics Group, Cavendish Laboratory, Madingley Road, \\ Cambridge CB3 0HE, UK.
}

\begin{abstract}
Systems of partial differential equations lie at the heart of physics. Despite this, the general theory of these systems has remained rather obscure in comparison to numerical approaches such as finite element models and various other discretisation schemes. There are, however, several theoretical approaches to systems of PDEs, including schemes based on differential algebra and geometric approaches including the theory of exterior differential systems [- [] and the so-called "formal theory" [4 built on the jet bundle formalism. This paper is a brief introduction to jet bundles focusing on the completion of systems to equivalent involutive systems for which power series solutions may be constructed order by order. We will not consider the mathematical underpinnings of involution (which lie in the theory of combinatorial decompositions of polynomial modules [2, 3]) nor other applications of the theory of jet bundles such as the theory of symmetries of systems of PDEs [6] or discretisation schemes based on discrete approximations to jet bundles [1].
\end{abstract}

\section{$1 \quad$ Fibre Bundles and Sections}

A bundle is a triple $(M, X, \pi)$, where $M$ is a manifold called the total space, $X$ is a manifold called the base space, and $\pi: M \rightarrow X$ is a continuous surjective mapping called the projection. Where no confusion can arise, we shall often find it convenient to denote the bundle either by its total space or its projection. A trivial bundle is a bundle whose total space $M$ is homeomorphic to $X \times U$, where $U$ is a manifold called the fibre. A bundle which is locally a trivial bundle is called a fibre bundle (an example of a fibre bundle which is not trivial is the Möbius band). In the following we shall only be concerned with trivial bundles.

We will denote the coordinates on the base manifold $x=\left\{x^{i}, i=1, \ldots, p\right\}$ where $p$ is the dimension of the base manifold and the coordinates on the fibre $u=\left\{u^{\alpha}, \alpha=1, \ldots, q\right\}$ where $q$ is the dimension of the fibre (when we consider jet bundles, we will need to extend this notation slightly). In the case of a trivial bundle, or in a local coordinate patch on a fibre bundle, the projection takes the simple form

$$
\pi:\left\{\begin{array}{l}
X \times U \rightarrow X \\
(x, u) \mapsto(x)
\end{array}\right.
$$

A section of a fibre bundle is a map

$$
\Phi_{f}:\left\{\begin{array}{l}
X \rightarrow X \times U \\
x \mapsto(x, f(x))
\end{array}\right.
$$

${ }^{1}$ e-mail: rcdb100@mrao.cam.ac.uk

${ }^{2}$ e-mail: c.doran@mrao.cam.ac.uk, http://www.mrao.cam.ac.uk/ cjld1/ 
such that $\pi \circ \Phi_{f}$ is the identity map on $X$. In other words, a section assigns to each point in $X$ a point in the fibre over that point. The graph of the function $f(x)$ is:

$$
\Gamma_{f}=\{(x, f(x)): x \in \Omega\} \subset X \times U
$$

where $\Omega$ is the domain of definition of $f$. We will find it convenient to refer to sections, functions and graphs interchangably.

\section{Jet Bundles}

We define a multi-index $J$ as a $p$-tuple $\left[j_{1}, j_{2}, \ldots, j_{p}\right]$ with $j_{i} \in \mathbb{N}$. The order of the multi-index $J$, denoted $|J|$ is given by the sum of the $j_{i}$. We will often find it more convenient to use a repeated-index notation for $J$. In this notation $J$ is represented by a string of $|J|$ independent coordinate labels, with $j_{i}$ copies of the $i$-th coordinate label. For example, if $p=3$ and the coordinates are labelled then $x, y$ and $z$ then the second order multi-indices in repeated index notation are $x x, x y, x z, y y, y z$ and $z z$. We introduce the special notation $J, i$ where $i$ is an independent coordinate label for the multi-index given by $\left[j_{1}, \ldots, j_{i}+1, \ldots, j_{p}\right]$. For example, $x y y, x=x x y y$.

If our independent variables are $x^{i}$ and our dependent variables are $u^{\alpha}$ then we introduce jet variables $u_{J}^{\alpha}$ where $J$ is a multi-index. Notice that we can put the jet variables of order $n$ in one-to-one correspondance with the derivatives of the dependent variables of order $n$. We will later introduce further structures that enable us to make a full correspondance between jet variables and derivatives.

Associated with these jet variables we introduce a set of Euclidean spaces $U_{i}$, whose coordinates are $u_{J}^{\alpha}$ with $|J|=i$. We call the space $M^{(1)}=X \times U \times U_{1}$ the first order jet bundle over the space $M=X \times U$. We now introduce the notation

$$
U^{(n)}=U \times U_{1} \times \cdots \times U_{n}
$$

and call the space $M^{(n)}=X \times U^{(n)}$ the $n$-th order jet bundle over $M$.

Example 1 Let $p=2$ and $q=1$. Label the independent variables $x$ and $y$ and the dependent variable $u$. The first order jet bundle, $M^{(1)}$, then has coordinates $\left(x, y, u, u_{x}, u_{y}\right)$, the second order jet bundle, $M^{(2)}$, has coordinates $\left(x, y, u, u_{x}, u_{y}, u_{x x}, u_{x y}, u_{y y}\right)$ and so on.

We will often consider a jet bundle as a bundle over a lower order jet bundle. We denote the natural projection between the $(m+n)$-th order jet bundle and the $n$-th order jet bundle as

$$
\pi_{n}^{m+n}: M^{(m+n)} \rightarrow M^{(n)}
$$

Note that although $M^{(m+n)}$ is a bundle over $M^{(n)}$ it is not a jet bundle over $M^{(n)}$, but rather a subset of such a bundle. 


\section{Differential Functions and Formal Derivatives}

A differential function is a smooth, real-valued function defined on $M^{(n)}$. We denote the algebra of differential functions defined on $M^{(n)}$ by $\mathcal{A}_{(n)}$. If $F \in \mathcal{A}_{(n)}$ then $F \in \mathcal{A}_{(m+n)}$ too, as the coordinates on $M^{(n)}$ are a subset of the coordinates on $M^{(m+n)}$. If the lowest order space on which $F$ is defined is $M^{(n)}$ then we will say that $F$ is an $n$-th order differential function. These will be used to describe sections of $M$ and differential equations.

The most fundamental maps between lower order and higher order jet bundles are provided by formal derivatives. A formal derivative operator $D_{i}$, called the formal derivative with respect to $x^{i}$, maps each differential function $F \in \mathcal{A}_{(n)}$ to a differential function $D_{i} F \in \mathcal{A}_{(n+1)}$ via

$$
D_{i} F=\frac{\partial F}{\partial x^{i}}+\sum_{\alpha=1}^{q} \sum_{J} \frac{\partial F}{\partial u_{J}^{\alpha}} u_{J, i}^{\alpha}
$$

It is convenient to extend the notation for formal and partial derivatives to encompass our multi-index notation:

$$
D_{J}=\left(D_{1}\right)^{j_{1}}\left(D_{2}\right)^{j_{2}} \ldots\left(D_{p}\right)^{j_{p}}
$$

and similarly for partial derivatives. Clearly if $F \in \mathcal{A}_{(n)}$ then $D_{J} F \in \mathcal{A}_{(n+|J|)}$.

\section{Prolongation of Sections}

If $\Gamma_{f} \subset M$ is a section defined by $u=f(x)$ where $f$ is a smooth function of $x$ then we can use the formal derivative to prolong it to a section $\Gamma_{f}^{(1)} \subset M^{(1)}$. The equations defining $\Gamma_{f}^{(1)}$ are simply $u^{\alpha}=f^{a}(x)$ and the equations found by applying each of the $D_{i}$ to $u^{\alpha}=f^{\alpha}(x)$ :

$$
\Gamma_{f}^{(1)}= \begin{cases}u^{\alpha} & =f^{\alpha}(x) \\ u_{i}^{\alpha} & =\partial_{i} f^{\alpha}(x)\end{cases}
$$

Similarly we can use the $D_{i}$ multiple times to prolong $\Gamma_{f}$ to a section $\Gamma_{f}^{(n)} \subset$ $M^{(n)}$ defined by the equations

$$
u_{J}^{\alpha}=\partial_{J} f^{\alpha}(x)
$$

where $J$ ranges over all multi-indices such that $0 \leqslant|J| \leqslant n$. We will sometimes talk about the $n$-th prolongation of a function $f(x)$, and write this prolongation as $f^{(n)}(x)$.

\section{Differential Equations and Solutions}

We intend to view systems of PDEs as geometric objects. In keeping with this programme, we will simply call such a system a "differential equation". An $n$-th order differential equation, $I_{\Delta}$, is a fibred submanifold of $M^{(n)}$. The differential equation is often stated as the kernel of a set of differential functions $\Delta_{\nu} \in \mathcal{A}_{(n)}$ :

$$
\Delta_{\nu}\left(x, u^{(n)}\right)=0, \quad \nu=1, \ldots, l
$$


We can map any system of partial differential equations onto such a submanifold, simply by replacing all of the derivatives of dependent variables by the corresponding jet variables. Indeed we have chosen our notation in such a way that this process is entirely transparent.

Example 2 The two dimensional wave equation

$$
\frac{\partial^{2} u}{\partial t^{2}}-\frac{\partial^{2} u}{\partial x^{2}}-\frac{\partial^{2} u}{\partial y^{2}}=0
$$

maps onto the submanifold of $M^{(2)}$ (with the obvious coordinates) determined by

$$
u_{t t}-u_{x x}-u_{y y}=0
$$

In keeping with our compact notations $x, u$ and $f$, we shall write

$$
\Delta\left(x, u^{(n)}\right)=\left(\Delta_{1}\left(x, u^{(n)}\right), \ldots, \Delta_{l}\left(x, u^{(n)}\right)\right) \in \mathcal{A}_{(n)}^{l}
$$

$\Delta$ is therefore a map from $M^{(n)}$ to $\mathbb{R}^{l}$. The differential equation $I_{\Delta}$ is the submanifold in which the map $\Delta$ vanishes:

$$
I_{\Delta}=\left\{\left(x, u^{(n)}\right): \Delta\left(x, u^{(n)}\right)=0\right\}
$$

A smooth solution of $I_{\Delta} \subset M^{(n)}$ is a smooth function $f(x)$ such that

$$
\Delta\left(x, f^{(n)}(x)\right)=0
$$

or, in terms of our geometric formulation

$$
\Gamma_{f}^{(n)} \subset I_{\Delta}
$$

Note that not every section of $M^{(n)}$ which lies entirely within $I_{\Delta}$ is a prolongation of a section of $M$ - the prolongation of a section of $M$ automatically respects the correspondence between jet variables and derivatives, whereas an arbitrary section of $M^{(n)}$ does not.

\section{Prolongation and Projection of Differential Equations}

The $k$-th prolongation of the differential equation

$$
I_{\Delta}=\left\{\left(x, u^{(n)}\right): \Delta_{\nu}\left(x, u^{(n)}\right)=0\right\} \subset M^{(n)}
$$

is

$$
I_{\Delta}^{(k)}=\left\{\left(x, u^{(n+k)}\right):\left(D_{J} \Delta_{\nu}\right)\left(x, u^{(n+k)}\right)=0\right\} \subset M^{(n+k)}
$$

where $J$ runs over all multi-indices up to order $k$.

Differential equations may be projected along the fibers onto lower order jet bundles. In general, this is a complicated procedure in local coordinates, but it is much easier if the differential equation is known to be the prolongation of a lower order system. For the remainder of this paper this will always be the case. 
Example 3 Let the differential equation $\mathcal{I} \subset M^{(2)}$ be defined by

$$
\mathcal{I}:\left\{\begin{aligned}
u_{z z}+u_{x y}+u & =0 \\
u_{x}-u & =0 \\
u_{y}-u^{2} & =0
\end{aligned}\right.
$$

The projection of $\mathcal{I}$ into $M^{(1)}$ is

$$
\pi_{1}^{2}(\mathcal{I}):\left\{\begin{array}{r}
u_{x}-u=0 \\
u_{y}-u^{2}=0
\end{array}\right.
$$

\section{Power Series Solutions}

A smooth solution of $\mathcal{I} \subset M^{(n)}$ in a neighbourhood of $x_{0}$ may be written as the power series

$$
u^{\alpha}=f^{\alpha}(x)=\sum_{|J|=0}^{\infty} \frac{a_{J}^{\alpha}}{J !}\left(x-x_{0}\right)^{J}
$$

for some constants $a_{J}^{\alpha}$. Here $J !=j_{1} ! j_{2} ! \ldots j_{p}$ ! and

$$
\left(x-x_{0}\right)^{J}=\prod_{i=1}^{p}\left(x^{i}-x_{0}^{i}\right)^{j_{i}}
$$

All we have to do is choose the values of the $a_{J}^{\alpha}$ so that $\Gamma_{f}^{(n)} \subset \mathcal{I}$. It will, however, prove to be easiest to fix the $a_{J}^{\alpha}$ by using the condition that $\Gamma_{f}^{(n+k)} \subset \mathcal{I}^{(k)}$ for all $k$ and working entirely at the point $x_{0}$. By applying the formal derivative repeatedly to the power series we can obtain power series expressions for each of the $u_{J}^{\alpha}(x)$. Evaluating each of these at $x_{0}$ shows that $a_{J}^{\alpha}=\left.u_{J}^{\alpha}\right|_{x_{0}}$. Therefore we require that

$$
D_{J} \Delta_{\nu}\left(x_{0}, a_{J}^{\alpha}\right)=0
$$

for all $J$. We have exchanged the solution of a set of partial differential equations for the solution of an infinite number of algebraic equations.

For a class of systems known as formally integrable differential equations we can construct a power series solution order by order. We first substitute the general form for the power series into each of the equations and evaluate at $x=x_{0}$. This gives us a set of algebraic equations for the $a_{J}^{\alpha}(x)$ with $|J| \leqslant n$. We then make a partition of the jet variables into parametric derivatives whose values we can choose and principal derivatives whose values are then fixed by the system, and solve for the latter in terms of the former. We then prolong the differential equation and repeat the process. This time the equations of order less than $n+1$ will automatically be satisfied by the previously chosen constants, and we will be left with a new set of equations $D_{i} \Delta_{\nu}\left(x_{0}, a_{J}^{\alpha}\right)=0$ for $|J|=n+1$. The nature of the formal derivative means that these equations will be linear. We may repeat the procedure to calculate ever higher terms in the power series. 


\section{Integrability Conditions}

If a differential equation is not formally integrable the solutions are subject to constraints which we call integrability conditions. These are extra equations that are differential rather than algebraic consequences of the equations $\Delta=0$. In other words, projecting the prolongation of a differential equation may not return the original equation but only a proper subset thereof:

$$
\pi_{n}^{n+k}\left(\mathcal{I}_{\Delta}^{(k)}\right) \varsubsetneqq \mathcal{I}_{\Delta} \subset M^{(n)}
$$

and so the order by order construction of a power series solution will be disrupted. To streamline the notation, we will write the $j$-th projection of the $k$-th prolongation of $\mathcal{I}$ as $\mathcal{I}_{j}^{(k)}$. For example, the expression above may be rewritten $\mathcal{I}_{k}^{(k)} \varsubsetneqq \mathcal{I}$.

Integrability conditions arise in two ways: through the differentiation of equations with order less than $n$ in $\mathcal{I} \subset M^{(n)}$, and through the effects of crossderivatives, as shown in the following example:

Example 4 Let $p=3$ with coordinates $x, y$ and $z$, and $q=1$ with coordinate $u$, and consider the differential equation

$$
\mathcal{I}:\left\{\begin{aligned}
u_{z}+y u_{x} & =0 \\
u_{y} & =0
\end{aligned}\right.
$$

which prolongs to

$$
\mathcal{I}^{(1)}:\left\{\begin{array}{r}
u_{x z}+y u_{x x}=0 \\
u_{y z}+u_{x}+y u_{x y}=0 \\
u_{z z}+y u_{x z}=0 \\
u_{x y}=0 \\
u_{y y}=0 \\
u_{y z}=0 \\
u_{z}+y u_{x}=0 \\
u_{y}=0
\end{array}\right.
$$

We see that the equations $u_{y z}=0$ and $u_{x y}=0$ substituted into the equation $u_{y z}+u_{x}+y u_{x y}=0$ imply that $u_{x}=0$. This is a first order equation and so forms part of $\mathcal{I}_{1}^{(1)}$ on projection. Hence $\mathcal{I}_{1}^{(1)} \varsubsetneqq \mathcal{I}$.

A differential equation $\mathcal{I} \subset M^{(n)}$ is formally integrable if $\mathcal{I}_{1}^{(k+1)}=\mathcal{I}^{(k)}$ for all $k$. Notice that to check for formal integrability requires an infinite number of operations, for integrability conditions may in general arise after an arbitrarily large number of prolongations.

\section{Involutive Differential Equations}

We now turn to the consideration of a subset of formally integrable differential equations known as involutive equations. Two facts make this class of equations interesting and useful. Firstly, it is possible to determine whether a given differential equation is involutive using only a finite number of operations. Secondly, 
for any differential equation it is possible to produce an involutive equation with the same solution space using only a finite number of operations.

There is a more systematic method for determining the integrability conditions that arise upon a single prolongation and projection. Let us look at the Jacobi matrix of $\mathcal{I}^{(1)} \subset M^{(n+1)}$. This matrix can be divided into four blocks:

$$
\begin{array}{|c|c|c|}
\hline \frac{\partial D_{i} \Delta_{\nu}}{\partial u_{J}^{\alpha}}, \quad 0 \leqslant|J| \leqslant n & \frac{\partial D_{i} \Delta_{\nu}}{\partial u_{J}^{\alpha}}, & |J|=n+1 \\
\hline \frac{\partial \Delta_{\nu}}{\partial u_{J}^{\alpha}}, & 0 \leqslant|J| \leqslant n & 0 \\
\hline
\end{array}
$$

We order the columns according to increasing $|J|$, and within each order by the first non-vanishing component of $\mathrm{J}$ (we will call this component the class of $\mathrm{J}$ ).

When we project the $\mathcal{I}^{(1)}$ into $M^{(n)}$ to form $\mathcal{I}^{(1)}$ we must include only those equations that are independent of the $u_{J}^{\alpha}$ with $|J|=n+1$. In other words we must include all those equations which have a full row of zeros in the right hand block. This clearly includes the equations corresponding to rows in the bottom part of the Jacobi matrix. However, if the upper right submatrix is not of maximal rank then we may be able to form integrability conditions. If for a row with all zeros in the right hand section we find that the left hand part is independent of the rows in the lower part of the matrix then there is indeed an integrability condition, which can be determined by performing the same operations on the full equations $D_{i} \Delta_{\nu}=0$.

We will call the system of equations defined by the upper right block of the Jacobi matrix the symbol of $\mathcal{I}$, and denote this Sym $\mathcal{I}$ :

$$
\operatorname{Sym} \mathcal{I}:\left\{\sum_{\alpha,|J|=n}\left(\frac{\partial \Delta_{\nu}}{\partial u_{J}^{\alpha}}\right) v_{J}^{\alpha}=0\right.
$$

where the $v_{J}^{\alpha}$ are a new set of variables, which we order in the same way as the the $u_{J}^{\alpha}$ when displaying the symbol as a matrix. Notice that the entries in the matrix of $\operatorname{Sym} \mathcal{I}$ are the coefficients of the highest order jet variables in the equations defining $\mathcal{I}^{(1)}$ as can be seen by comparison with the formal derivative.

Comparison of the ranks of $\mathcal{I}, \mathcal{I}^{(1)}$ and $\operatorname{Sym} \mathcal{I}^{(1)}$ will enable us to determine if an integrability condition will occur on a single prolongation and projection. There is an integrability condition if $\operatorname{rank} \mathcal{I}_{1}^{(1)}>\operatorname{rank} \mathcal{I}$, or equivalently if $\operatorname{dim} \mathcal{I}_{1}^{(1)}<\operatorname{dim} \mathcal{I}$. Furthermore from inspection of the Jacobi matrix of $\mathcal{I}^{(1)}$

$$
\operatorname{rank} \mathcal{I}_{1}^{(1)}=\operatorname{rank} \mathcal{I}^{(1)}-\operatorname{rank} \operatorname{Sym} \mathcal{I}^{(1)}
$$

We can thus systematically determine if integrability conditions arise from a single prolongation, and if necessary find the new equations.

Henceforth we will always consider the row echelon form of the symbol. We call Sym $\mathcal{I}$ involutive if

$$
\operatorname{rank} \operatorname{Sym} \mathcal{I}^{(1)}=\sum_{k} k \beta_{k}
$$

where $\beta_{k}$ is the number of rows of class $k$ in $\operatorname{Sym} \mathcal{I}$.

For a row of class $k$ we call the variables $x^{1}, x^{2}, \ldots, x^{k}$ multiplicative variables. We now consider prolonging each equation by its multiplicative variables 
only. The equations obtained in this manner will be independent as they will have distinct pivots in $\operatorname{Sym} \mathcal{I}^{(n)}$. As there are $\beta_{k}$ equations of class $k$ and each has $k$ multiplicative variables then this means there will be at least $\sum k \beta_{k}$ independent equations of order $n+1$ in $\mathcal{I}^{(n+1)}$. If $\operatorname{Sym} \mathcal{I}$ is involutive then we obtain all the independent equations of order $n+1$ in this manner. The equations obtained from the other prolongations required to prolong $\mathcal{I}$ to $\mathcal{I}^{(1)}$ will thus be dependent, of lower order, or both.

The importance of involutive symbols arises from the following theorem which provides a criterion for involution that can be tested in a finite number of operations:

Theorem $1 \mathcal{I}$ is involutive if and only if $\operatorname{Sym} \mathcal{I}$ is involutive and $\mathcal{I}_{1}^{(1)}=\mathcal{I}$.

\section{Cartan-Kuranishi Completion}

The central theorem of the theory of involutive sytems of differential equations is the Cartan-Kuranishi theorem:

Theorem 2 For every differential equation $\mathcal{I}$ there are two integers $j, k$ such that $\mathcal{I}_{j}^{(k)}$ is an involutive equation with the same solution space.

The Cartan-Kuranishi completion algorithm is a straightforward application of the two previous theorems:

input $\mathcal{I}$

repeat

while Sym $\mathcal{I}$ is not involutive repeat $\mathcal{I}:=\mathcal{I}^{(1)}$

while $\mathcal{I} \neq \mathcal{I}_{1}^{(1)}$ repeat $\mathcal{I}:=\mathcal{I}_{1}^{(1)}$

until Sym $\mathcal{I}$ is involutive and $\mathcal{I}=\mathcal{I}_{1}^{(1)}$

output involutive $\mathcal{I}$

Therefore, given a differential equation we may first complete it to an involutive system (if it is not already involutive) and then construct a power series solution order by order using the algorithm described earlier.

\section{Conclusion}

Although the algorithms described in this paper may be used to construct formal solutions to systems of partial differential equations, they suffer from several shortcomings in practice. Firstly, there is the problem of setting the values of the parametric derivatives. Typically, these must be calculated from the values of functions on submanifolds of the base space or from values of those functions at the points of a lattice within the base space. Secondly, many terms of the power series must be calculated to provide solutions of comparable accuracy to those produced by discretisation schemes, and the symbolic manipulations involved rapidly become computationally intensive as the order increases. Thirdly, there is the problem of the convergence of the series. A promising approach to the circumvention of these difficulties is the use of a hybrid method that first uses a discretisation scheme to calculate values at lattice points, uses these values to determine approximate values of the parametric derivatives and then constructs 
power series about each of the points and smoothly joins them together using a functional interpolation scheme. This method is currently being implemented.

Completion to involution and the construction of power series solutions are far from the only applications of the jet bundle formalism. As mentioned in the introduction, jet bundles also provide the natural setting for the analysis of the symmetry groups of systems of PDEs and of the variational symmetries of Lagrangian systems (which are linked by Noether's theorem to conservation laws). Symmetry analysis is also closely related to the construction of solutions possessing specified symmetries. Unfortunately, these fascinating and important subjects are beyond the scope of the current paper.

\section{References}

[1] J. Marsden, G. Patrick and S. Shkoller, Multisymplectic Geometry, Variational Integrators, and Nonlinear PDEs, Comm. Math. Phys., vol. 199, pp. 351-395, 1998.

[2] J. Calmet, M. Hausdorf and W. Seiler, A Constructive Introduction to Involution, Proc. ISACA 2000, World Scientific, Singapore, 2000.

[3] W. Seiler, A Combinatorial Approach to Involution and $\delta$-Regularity, Preprint, Universitat Mannheim 2000

[4] W. Seiler, Analysis and Application of the Formal Theory of Partial Differential Equations, School of Physics and Materials, Lancaster University, 1994.

[5] Y. Choquet-Bruhat, C. DeWitt-Morette and M. Dillard-Bleick, Analysis, Manifolds and Physics Part 1, North-Holland, 1982.

[6] P. Olver, Applications of Lie Groups to Differential Equations, SpringerVerlag New York, 2000. 